\title{
LA SEXUALIDAD EN ADULTOS MAYORES
}

The sexuality in older adults

\section{Susana Alvarado Alfaro', a,b}

${ }^{1}$ Facultad de Enfermería de la Universidad Nacional San Luis Gonzaga de Ica, Perú ${ }^{a}$ Magister en Administración y Planificación de la Educación Superior. ${ }^{b}$ Licenciada en Enfermería

\section{RESUMEN}

Objetivos: Conocer la sexualidad en los adultos mayores, a partir de una revisión de investigaciones previas, realizadas en el ámbito internacional; Material y métodos: investigación no experimental, cualitativa, descriptiva retrospectiva, transversal. Se han utilizado como instrumentos de recojo de información fichas, y como técnicas la observación, el análisis, y la síntesis, el método seleccionado fue el deductivo. Se revisaron 15 investigaciones realizadas en diversos países referidas a la sexualidad del adulto mayor. Resultados: Se han encontrado algunas descripciones sobre actitudes sexuales, en diversos pueblos con anterioridad al año 100 Antes de Cristo, y recién en el siglo XX se empieza a investigar la sexualidad desde el punto de vista científico, con los aportes de Balzac, Jung (1969) y Sigmund Freud (1856-1939). Existen factores y mitos que afectan y están relacionados con la sexualidad del adulto mayor. La existencia de un declive gradual con la edad, en la actividad e interés sexual, en el caso de los varones se produce a partir de los 60 años, la actividad sexual más frecuente son las caricias y tocamientos, seguidas del coito, también se observa una mayor actividad sexual en las mujeres mayores que han tenido intensa actividad sexual en la juventud. Un alto número (74\% hombres, $42 \%$ mujeres) practican la masturbación, la relevancia del sexo decae a partir de los 56 años de edad, llegando a considerarse como nada o poco importante en el $57 \%$ de las personas mayores de 65 años. Conclusiones: La sexualidad incluye todas las formas de expresión, desde la aproximación, el tacto, la intimidad emocional, la compañía, la masturbación y no solamente el coito. Es un aspecto importante que en la vejez debe continuar siendo una fuente de placer y no una fuente de inquietud y frustración.

Palabras clave: Sexualidad en adultos mayores.

\section{SUMMARY}

Objectives: Learn about sexuality in older adults, based on a review of previous research carried out at the international level; Material and methods: research non-experimental, qualitative, descriptive retrospective, cross-sectional. They have been used as instruments of pick up information sheets, and as techniques of observation, analysis, and synthesis, the method selected was the deductive. Reviewed 15 research projects in different countries concerning the sexuality of the elderly. Results: Found some descriptions about sexual attitudes, by diverse peoples, prior to the year 100 A.C, and only in the 20th century is beginning to investigate the sexuality from the scientific point of view, with the contributions of Balzac, Jung (1969) and Sigmund Freud (1856-1939). There are factors and myths that affect and are related to the sexuality of the elderly, various studies show it. The existence of a gradual decline with age, activity and sexual interest, in the case of males is produced from the age of 60 , the most frequent sexual activity are caressing and touching, followed by sexual intercourse, also observed a greater sexual activity in older women who have had intense sexual activity in young people. A high number (74\% men, $42 \%$ women) they practice masturbation, the relevance of sex decays from the 56 years of age, to be considered as nothing or little important in $57 \%$ of seniors aged 65 . Conclusions: Sexuality includes all forms of expression, from the approximation, touch, emotional intimacy, the company, masturbation and not only sexual intercourse. It is important that, in old age, must continue to be a source of pleasure and not a source of concern and frustration.

Keywords: sexuality in older adults. 


\section{INTRODUCCION}

En el mundo actual las personas viven cada vez más años y con mejor estado de salud, lo cual se evidencia en el incremento de las tasa de esperanza de vida, como consecuencia del desarrollo tecnológico, científico, clínico, y otros campos del saber científico. Sin embargo, aunque la atención del adulto mayor ha alcanzado logros notables, en el tratamiento de su sexualidad persisten actitudes retrógradas que son similares a las existentes desde siglos anteriores, y que tienden a rechazar o burlarse, y en el mejor de los casos ignorar, la existencia de necesidades sexuales en el adulto mayor. A mucha gente se les hace difícil pensar que los adultos mayores, sean hombres o mujeres, tengan sentimientos, necesidades y relaciones de tipo sexual, y esto viene dado por los estereotipos sexuales existentes. En muchos casos o circunstancias, tales como trastornos de la salud, pérdida del cónyuge, etc., se crea una base física y social real que justifica la inexistencia de actividad sexual, pero no quiere decir que en estas personas no continúe la existencia del interés sexual (1). Subsisten todavía prejuicios socioculturales respecto a la sexualidad del adulto mayor, y puede tener dos explicaciones (2): La primera, es la incorrecta asociación entre la sexualidad y la reproducción, mediante la cual se considera solo normal la actividad sexual en época reproductiva, y por tanto, los ancianos no tienen por qué practicarla, la segunda es la existencia del prejuicio de que ser viejo es igual a enfermo (3).

Las primeras consideraciones sobre la sexualidad datan desde hace aproximadamente 5000 años, si bien existe información limitada sobre la descripción y actitudes sexuales con anterioridad al año 100 Antes de Cristo, es en el siglo $X X$ cuando se empieza a investigar la sexualidad desde el punto de vista más científico (4) a partir de una visión más positiva de la sexualidad, a cargo de Balzac, Jung (1969) y Sigmund Freud (1856-1939).
Los filósofos señalan que el hombre es por sí mismo un misterio, y que su sexualidad participa de esta trascendencia, y se dice que es por ello que ni las más sofisticadas investigaciones conseguirán nunca explorarla totalmente, salvo que se integren en la antropología filosófica o teológica para enriquecer la capacidad analítica. La filosofía espiritualista moderna insiste en la totalidad del hombre, exalta su poder de autodeterminación, subraya la tendencia de la persona hacia los valores, dentro de este marco, la sexualidad aparece como la condición y hasta el lugar de la experiencia problemática y metafísica. La naturaleza sexuada del hombre y de la mujer se convierte en un medio, con el cual ambos intercambian sus riquezas respectivas $y$ sienten que la intencionalidad presente en su acto se eleva a través de su genitalidad a un nivel superior (5) Nuttin, manifiesta que "el objeto sexual es infinitamente más rico que el cuerpo objetivo; en él está la persona revestida de misterio y de la atracción que ejerce su personalidad íntima". El análisis filosófico de la sexualidad todavía no ha igualado al progreso de las ciencias positivas, sin embargo se prevé que en las próximas décadas llegue a ser un campo de fecunda investigación para los personalistas. La nueva cultura de la sexualidad, con el aporte científico, filosófico y teológico; puede desarrollarse de forma distinta a la que ahora está en auge y ayudar a los adultos a disfrutar de una dimensión amenazada por la persistente hipoteca del materialismo. Algo muy importante es la reflexión acerca del valor educativo de los estudios sobre el arco vital de la persona para mostrar que la evolución ocupa toda la existencia del individuo. El mérito de hacer resaltar los estadios posteriores a la adolescencia corresponde a Jung, seguido por Levinson "fundador del estudio del desarrollo adulto", y continuado por Erikson, a quien se debe la descripción de algunos estadios posjuveniles, que fue ampliada por los miembros de su escuela, los psicólogos estadounidenses 
Kohlberg, Gould, y Lóvinger y por los alemanes Thomae, y Lehr.

Durante mucho tiempo la psicología, por influjo de Freud, dio a entender que el crecimiento terminaba con la adolescencia, esta influencia condiciono a la pedagogía y a la educación, circunscribiéndolo a la infancia y pubertad. Las últimas investigaciones han involucrado vida adulta y la ancianidad, refutando la doctrina freudiana (5).

Siguiendo los conceptos expuestos, la educación sexual plantea dos consideraciones: La primera se refiere a los benéficos, efectos que sobre el estadio posterior tiene una formación bien cimentada en el anterior, C. Bühler, muestra la incapacidad de muchas personas para orientar positivamente sus instintos debido a problemas de relación surgidos en los primeros años con sus padres; L. Ancona, señala que en la infancia se fijan presupuestos esenciales para el futuro amor conyugal. La segunda consideración se refiere a los adultos en situaciones difíciles, que tienen relación con la infancia y los traumas psicológicos posteriores; o por la falta de proyectos para el futuro, la forma que la vida se hace apática en la rutina cotidiana (5).

El objetivo fue conocer la sexualidad en los adultos mayores, a partir de una revisión de investigaciones previas, realizadas en el ámbito internacional.

\section{MATERIAL Y METODOS}

Investigación cualitativa, descriptiva retrospectiva y transversal. Se han utilizado como instrumentos recolectores de información fichas, y como técnicas la observación, el análisis, y la síntesis; método deductivo. La población ha sido la diversidad de trabajos, y publicaciones sobre la sexualidad en adultos mayores, de las cuales se obtuvo como muestra 15 investigaciones realizadas en diversos países. El proceso de recolección comprendió la búsqueda documental, y electrónica.

\section{RESULTADOS}

De la revisión teórica y producto del análisis se han encontrado algunas descripciones sobre actitudes sexuales, en diversos pueblos con anterioridad al año 100 Antes de Cristo, y recién en el siglo $X X$ se empieza a investigar la sexualidad desde el punto de vista más científico, con los aportes de Balzac, Jung (1969) y Sigmund Freud (1856-1939).

Se ha establecido que existen factores y mitos que afectan y están relacionados con la sexualidad del adulto mayor, los estudios de Kinsey (1948), Kaiser (1996), Schneider (1994), Schiavi-Rehman (1995), Nieto (1995), Dello (1998), Mc Kinlay (1999), Bobes (2000), Moioli (2005), Ballone (2002), Cibeles (2004), Kaplan y Sadock, Piñango y Montalvo (2009), y otros, evidencian: La existencia de un declive gradual con la edad, en la actividad e interés sexual tanto en varones como en mujeres, en el caso de los varones se produce a partir de los 60 años. La actividad sexual disminuye conforme avanza la edad, y la actividad sexual más frecuente son las caricias y tocamientos, seguidas del coito, también se observa una mayor actividad sexual en las mujeres mayores que han tenido intensa actividad sexual en la juventud, un alto número (74\% hombres, $42 \%$ mujeres) practican la masturbación, la relevancia del sexo decae a partir de los 56 años de edad, llegando a considerarse como nada o poco importante en el $57 \%$ de las personas mayores de 65 años. La sexualidad dura toda la vida y es beneficiosa para la salud, los ancianos tienen poca privacidad para mantener relaciones, muchos de ellos viven con familiares, hijos, nietos, y la actividad sexual frecuentemente se ve limitada por la ausencia de pareja y por el estado de salud.

\section{DISCUSION}

Existen conceptos equivocados que plantean que la actividad sexual debe desaparecer en la edad avanzada, como una culminación fisiológica inevitable y por lo tanto, la 
sexualidad después de los 60 años, no es natural, fisiológica, moral, o socialmente bien vista. De ahí que esta idea de muerte sexual se convierta para muchas personas de ambos sexos en un factor de ansiedad cuando llega a la edad madura y ve aproximarse esta etapa crítica, lo que provoca el inicio de trastornos en la función sexual de índole puramente psíquico, en concordancia con Echeverri (6) y Zuleta (7)

Masters y Johnson, señalan que el envejecimiento tiende a disminuir la actividad sexual, pero no a terminar con ella, la forma más eficaz para mantener la actividad sexual en la vejez es practicar el sexo con frecuencia durante la juventud y en la madurez. La asociación que existe entre sexualidad - coito -capacidad reproductiva, y habiendo perdido algunas de esas posibilidades y disminuido otras, lleva a que la sexualidad en el periodo de la adultez mayor sea comúnmente negada. De acuerdo con Carnivall (8), esto se traduce en sufrimiento y peor calidad de vida. Desde Freud muchos autores han desplegado teorías al respecto, analizando la sexualidad, y sus manifestaciones.

Aunque sostenemos enfáticamente la diferencia entre sexualidad y genitalidad, cuesta mucho no caer en sistematizaciones que pretenden semejanzas, como si la sexualidad se expresara exclusivamente por medios genitales.

En cuanto al enfoque filosófico y sociológico dado por Galli, la autora de este artículo se sitúa en la misma posición que fundamenta su teoría en el concepto de persona, es decir, entre los seguidores de una ontología fuerte y de una actitud positiva sobre la sexualidad en el adulto mayor.

\section{CONCLUSIONES}

1. La sexualidad incluye todas las formas de expresión, desde la aproximación, el tacto, la intimidad emocional, la compañía, la masturbación y no solamente el coito. Es un aspecto importante en la vejez, fuente de placer y no debe ser de frustración.

2. Las enfermedades, la viudez y el temor son los aspectos fundamentales que atentan contra la sexualidad de los adultos mayores, así mismo la falta de privacidad, la jubilación y sus secuelas, la monotonía, los hábitos tóxicos, la falta de comunicación, pérdida del deseo, y el filocentrismo.

3. Se han encontrado numerosos reportes acerca de que en los adultos mayores se mantiene la actividad sexual en un porcentaje elevado de ellos, y que se mantiene vivo el interés en el sexo, todo lo cual nos hace pensar que la sexualidad en esta época no es una utopía.

4. La violación de la privacidad se plantea en primer lugar dentro de toda la lista de factores, sin embargo muchas veces, los adultos mayores la facilitan (deben ocuparse de hijos y nietos, viven por lo general con 3 generaciones, y comparten sus habitaciones con otras personas).

\section{Correspondencia}

Mg. Susana Alvarado Alfaro

Correo Electrónico: Susanasa11@hotmail.com

\section{REFERENCIAS BIBLIOGRAFICAS}

1. Kolodny R. Tratado de medicina sexual, cap. 5 La Habana, Cuba: Edit. Científico Técnica, 1985, p. 84-5 (Ed. Revolucionaria).

2. Noriega D. Wally T. Sex Soc 1(1):14-15.

3. Heleger S. Sexuality and aging. Bristh Journal of Sexual Medicine Brit J Sex Med 1978; 32(5):16-19.

4. Blázquez D. "Los Derechos de las personas mayores" Ed. Paperback, Madrid, España. Marzo 26, 2009

5. Galli, N., Educación sexual y cambio cultural, Herder, Barcelona, España. 1984. 
6. Echeverri F. Familia y Vejez en Colombia.

Perspectivas año 2000. Santafé de

Bogotá: Universidad Nacional de Colombia y Colciencias, 1990.

7. Zuleta C. Factores psicológicos intervinientes en la calidad de vida de personas en la etapa de la vejez. Colombia. 2010. [Internet] Disponible en www.monografias.com.

8. Carnivall D. Tratado de geriatría y gerontología. 3 ed. México, DF: Interamericana, 2008:20-32.

Recibido: 23/02/14

Aprobado para Publicación: 29/05/14 